\title{
The cytoprotective role of gemcitabine-induced autophagy associated with apoptosis inhibition in triple-negative MDA-MB-231 breast cancer cells
}

\author{
MING CHEN $^{1 *}$, MENGYE HE $^{1 *}$, YINJING SONG $^{2}$, LUOQUAN CHEN $^{2}$, \\ PENG XIAO $^{2}$, XIAOPENG WAN $^{2}$, FENG DAI $^{2}$ and PENG SHEN ${ }^{1}$ \\ ${ }^{1}$ Department of Medical Oncology, The First Affiliated Hospital, College of Medicine; \\ ${ }^{2}$ Institute of Immunology, Zhejiang University, Hangzhou 310003, P.R. China
}

Received September 12, 2013; Accepted April 28, 2014

DOI: $10.3892 /$ ijmm.2014.1772

\begin{abstract}
Triple-negative breast cancer (TNBC), which is estrogen receptor (ER)-negative, progesterone receptor-negative and is also negative for HER2 expression, remains a great clinical challenge due to its strong resistance to chemotherapy at the late stage of treatment and relatively unfavorable prognosis. Gemcitabine has been approved by the FDA/SFDA for use as a first-line therapeutic drug against advanced or metastatic breast cancer. Therefore, the clarification of the mechanisms underlying gemcitabine-acquired resistance is of particular importance for the optimal management of TNBC. A number of studies have revealed that autophagy, which has been found to protect cancer cells from anti-cancer drug-induced death, may contribute to the development of drug resistance. However, the association between autophagy and gemcitabine treatment in TNBC cells has yet to be defined. Our study clearly demonstrates that gemcitabine is able to induce mTOR-independent autophagy in human triple-negative MDA-MB-231 breast cancer cells. In addition, we demonstrate that autophagy protects MDA-MB231 cells from gemcitabine-induced cell growth inhibition and apoptosis, indicating that gemcitabine can activate autophagy to impair the sensitivity of MDA-MB-231 cells. Furthermore, as shown by our results, the inhibition of gemcitabine-induced autophagy by chloroquine shifts the expression of the p53 protein, Bcl-2 family proteins and the relative Bax/Bcl-xL ratio in favor of promoting apoptosis. These results reveal that the inhibition of apoptosis may be one of the mechanisms of
\end{abstract}

Correspondence to: Professor Peng Shen, Department of Medical Oncology, The First Affiliated Hospital, College of Medicine, Zhejiang University, 79 Qingchun Road, Hangzhou 310003, P.R. China

E-mail: shenp@zju.edu.cn

\section{*Contributed equally}

Key words: autophagy, apoptosis, MDA-MB-231 cell, gemcitabine, resistance, triple-negative breast cancer autophagy-induced cytoprotection in gemcitabine-treated MDA-MB-231 cells. The apoptotic and autophagic processes constitute a mutual inhibition system and jointly seal the fate of TNBC cells that are exposed to gemcitabine. Thus, our study suggests that the combination of an autophagic inhibitor and gemcitabine as a therapeutic strategy may represent a promising approach with greater clinical efficacy for patients with TNBC. However, extended preclinical trials are required to further determine the positive effects of the inhibition of autophagy on the efficacy of gemcitabine.

\section{Introduction}

Breast cancer is the most prevalent malignancy and was expected to be the second leading cause of cancer-related mortality among women in the United States in 2013 (1). Triplenegative breast cancer (TNBC), accounting for approximately $15 \%$ of breast cancer cases, is an immunohistochemicallydefined phenotype and is estrogen receptor (ER)-negative, progesterone receptor (PR)-negative and is also negative for HER2 expression (2). Currently, TNBC remains a tremendous clinical challenge due to its aggressive behavior and relatively unfavorable prognosis (3). It is not only associated with an early relapse and a high risk of visceral and central nervous system metastases, but also with an early mortality during the first 3-5 years of follow up (4-7). Since TNBC is refractory to endocrine therapies, such as tamoxifen and aromatase inhibitors, as well as current HER2-targeted therapies, such as trastuzumab, chemotherapy remains the mainstay of treatment for patients with TNBC (2). Therefore, the improvement of chemotherapeutic efficacy is of particular importance to the optimal management of TNBC.

Gemcitabine is a deoxycytidine analogue, which competes with deoxycytidine triphosphate for incorporation into DNA, and thus inhibits DNA synthesis mainly in its triphosphate form (8). It has been used in a wide spectrum of malignancies, both as a single agent and in combination with other cytotoxic drugs. As a single agent, the response rates of gemcitabine range from 14 to $37 \%$ as a first-line therapy for advanced breast cancer and $12-30 \%$ in patients previously treated with anthracyclines and/or taxanes (9). In combination with taxanes and/ 
or anthracyclines as doublets or triplets, gemcitabine has been determined as a first-line therapy in clinical practice for the treatment of patients with metastatic breast cancer, achieving overall response rates ranging from 39 to $75 \%$ (10-12). However, intrinsic and acquired resistance to gemcitabine is common, whereas the molecular mechanisms underlying chemoresistance to gemcitabine are not yet fully understood (13).

Autophagy is an evolutionarily well-conserved lysosomal degradation pathway that maintains cytoplasmic homeostasis by eliminating protein aggregates and damaged organelles (14). It is characterized by the formation of double-membraned autophagosomes that engulf cytoplasmic components and eventually fuse with lysosomes to form autolysosomes for the degradation of the contents (15). Considering the role of autophagy in helping cells to adapt to starvation or unfavorable stress conditions (e.g., hypoxia, radiation therapy and cytotoxic drugs), autophagy is usually identified as a cellular pro-survival mechanism (16). By contrast, under certain circumstances, autophagy may also lead to cell death directly, causing autophagic cell death or type II programmed cell death (PCD; type II PCD), which is distinct from apoptosis (type I PCD) or necrosis (type III PCD) (17-20).

As regards the connections between the apoptotic and autophagic processes, a complex interplay between them has come to light, taking into account that numerous genes, such as p53, the death-associated protein kinase (DAPK) family and Bcl-2 family members, are shared between these two response machineries. This suggests that apoptosis and autophagy jointly seal the fate of cells (21).

A number of studies have demonstrated that gemcitabine induces autophagy in pancreatic cancer cells and the modulation of autophagy may improve the efficacy of gemcitabine (22-25). However, whether autophagy is associated with gemcitabine treatment for TNBC remains unknown. In the current study, we aimed to explore whether gemcitabine treatment induces autophagy in triple-negative MDA-MB-231 breast cancer cells, and establish the function of such inducible autophagy in the therapeutic efficacy of gemcitabine. Moreover, the possible interplay between autophagy and apoptosis in triple-negative MDA-MB-231 cells is also explored.

\section{Materials and methods}

Reagents and antibodies. The chemicals used in the present study were the following: gemcitabine (G6423), chloroquine diphosphate salt (C6628) and monodansylcadaverine (MDC; 30432) (all from Sigma-Aldrich, St. Louis, MO, USA). The Annexin V FITC Apoptosis Detection kit (88-8005) and the propidium iodide (PI) staining solution (00-6990) were from eBioscience, Inc. (San Diego, CA, USA). The primary antibodies were the following: anti-LC3B antibody produced in rabbit (L7543; Sigma-Aldrich), beclin 1 antibody (NB500-249; Novus Biologicals, Littleton, USA), anti-Bax rabbit polyclonal antibody (AB20073a), anti-Bcl-2L1 rabbit polyclonal antibody (AB20306a) (both from Life Science Products \& Services), anti-p53 (WBL, K0181-3) and mTOR rabbit antibody (\#2972; Cell Signaling Technology, Danvers, MA, USA). The secondary antibodies, HRP-conjugated goat anti-rabbit (sc-2357) and goat anti-mouse IgG (sc-2371), were obtained from Santa Cruz Biotechnology, Inc. (Santa Cruz, CA, USA).
Cell culture. The monolayer culture of MDA-MB-231 cells [obtained from the American Type Culture Collection (ATCC), Manassas, VA, USA] was maintained in L-15 medium supplemented with $10 \%$ fetal bovine serum, $100 \mu \mathrm{g} / \mathrm{ml}$ streptomycin and $100 \mathrm{U} / \mathrm{ml}$ penicillin in a humid incubator without $\mathrm{CO}_{2}$ at $37^{\circ} \mathrm{C}$.

MTT assay. Cells seeded in 96-well plates were allowed to attach overnight, and treated with various concentrations of the reagents for the indicated periods of time. Subsequently, $20 \mu \mathrm{l}$ MTT $(5 \mathrm{mg} / \mathrm{ml})$ were added to each well for $4 \mathrm{~h}$ of incubation. The supernatant was then removed and $150 \mu 1$ DMSO were added to each well to dissolve the blue-purple formazan crystals. The plates were agitated for $15 \mathrm{~min}$. Absorbance values at $490 \mathrm{~nm}$ were detected with a model 680 MicroPlate Reader (Bio-Rad, Hercules, CA, USA).

Flow cytometry with Annexin V-FITC (AV-FITC)/PI staining. The cells were seeded at $4 \times 10^{5}$ cells/well in 6-well plates and allowed to attach overnight. The cells were then treated for the indicated periods of time and were then collected and dyed with AV-FITC and PI according to the instructions of the manufacturer. Cell viability was analyzed by flow cytometry (BD FACSCalibur; BD Biosciences, San Jose, CA, USA).

Western blot analysis. The cells were treated for the indicated periods of time. Both the detached and attached cells were collected, and whole cell lysates were prepared. Approximately $30 \mu \mathrm{g}$ total proteins were separated by SDS-PAGE with a proper concentration and transferred onto PVDF membranes (Bio-Rad). The PVDF membranes were blocked by $5 \%$ non-fat dry milk for $90 \mathrm{~min}$ at room temperature, and were then incubated with primary antibodies overnight with light agitation at $4{ }^{\circ} \mathrm{C}$ and secondary antibodies for $2 \mathrm{~h}$ at room temperature. Visualization was carried out with electrochemiluminescence (ECL; Bio-Rad).

Visualization of MDC-labeled vacuoles. The cells were cultured on coverslips in 24-well plates and treated for the indicated periods of time. Subsequently, fresh medium-containing MDC $(50 \mu \mathrm{mol} / 1)$ was added to the plates for $15 \mathrm{~min}$. Subsequently, the cells were fixed using a freshly-prepared $4 \%$ formaldehyde solution for $10 \mathrm{~min}$ at room temperature. The specimens were analyzed under a BX61W1-FV1000 confocal microscope (Olympus, Tokyo, Japan).

EGFP-LC3 plasmid transfection. The EGFP-LC3 plasmids were transiently transfected into the cells using Lipofectamine $2000^{\mathrm{TM}}$ (Invitrogen Life Technologies, Carlsbad, CA, USA) according to the instructions of the manufacturer. After $24 \mathrm{~h}$, the cells were treated with the chemicals for the indicated periods of time, and then fixed with $4 \%$ formaldehyde solution for $10 \mathrm{~min}$ at room temperature. The fluorescence of EGFP was analyzed using a BX61W1-FV1000 confocal microscope (Olympus). The number of $\mathrm{LC} 3-\mathrm{II}^{+}$puncta was counted manually in confocal images from random fields to quantify the EGFP-LC3-tagged autophagosomes.

Transmission electron microscopy (TEM). The treated cells were fixed for $1 \mathrm{~h}$ in ice-cold 2.5 glutaraldehyde and $1 \%$ 
A

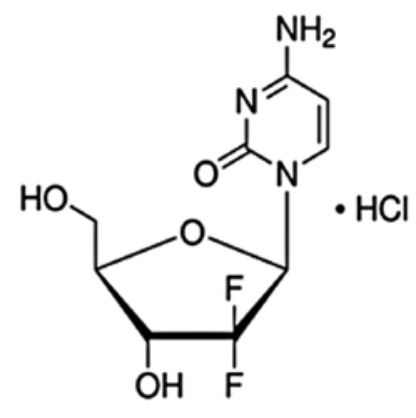

B

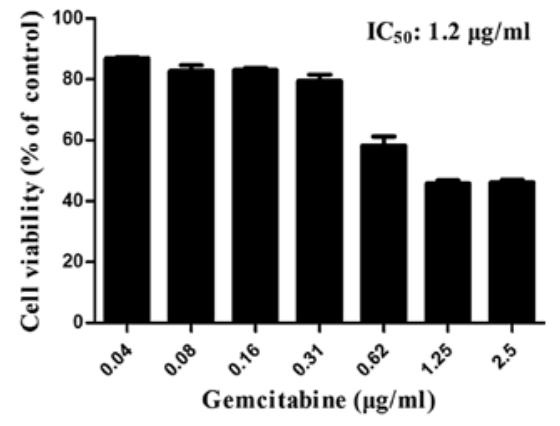

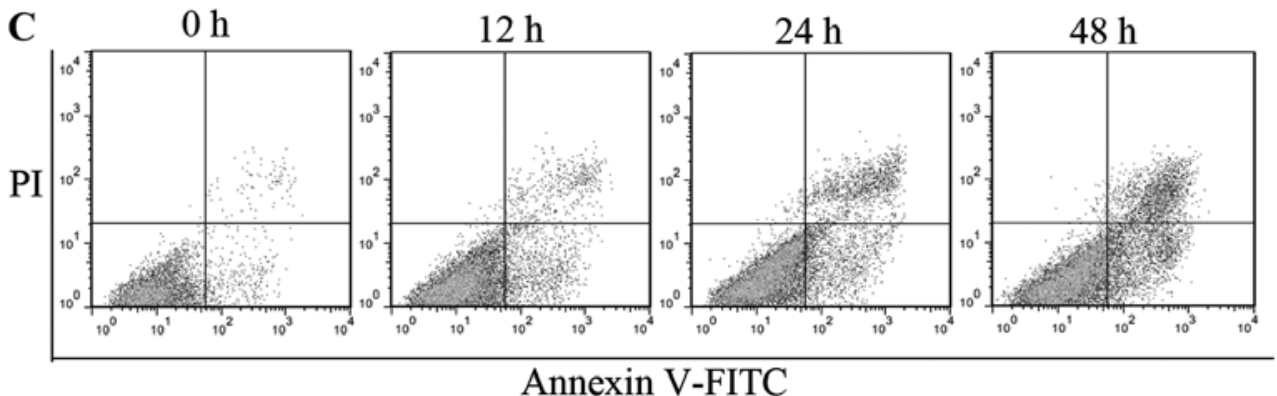

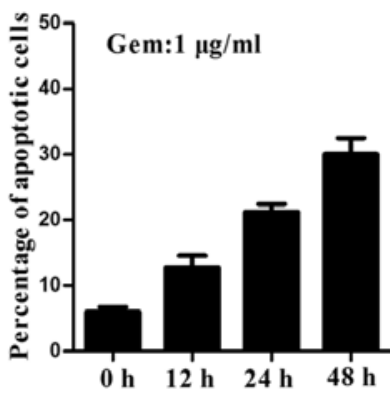

Figure 1. Gemcitabine decreases cell viability and induces apoptosis in MDA-MB-231 breast cancer cells. (A) Chemical structure of 2'-deoxy-2',2'-difluorocytidine (gemcitabine hydrochloride). (B) Cell viability following treatment with gemcitabine $(0.04-2.5 \mu \mathrm{g} / \mathrm{ml})$ for $48 \mathrm{~h}$ was determined by MTT assay in MDA-MB-231 cells. Results are expressed as the relative percentage of each group compared with the control. The results [means \pm standard error (SE)] were from 3 independent experiments. (C) Apoptosis was measured by Annexin V-FITC (AV-FITC)/propidium iodide (PI) staining and flow cytometry following treatment with gemcitabine $(1 \mu \mathrm{g} / \mathrm{ml})$ for $0 \mathrm{~h}$ (untreated control) or 12, 24 and $48 \mathrm{~h}$. The scatter plots and statistical analysis of Annexin V-FITC and PI uptake are shown. The results (means $\pm \mathrm{SE}$ ) were from 3 independent experiments.

osmium tetroxide for a further $1 \mathrm{~h}$. The samples were stained with $4 \%$ uranyl acetate for $30 \mathrm{~min}$, dehydrated through a graded series of ethanol solutions (ranging from 50 to 100\%) and embedded in Epon resin. Ultrathin sections $(120 \mathrm{~nm})$ were post-stained with uranyl acetate and lead citrate, and were then examined under a TECNAI 10 transmission electron microscope (Philips, Amsterdam, The Netherlands) at $60 \mathrm{kV}$.

RNA interference. The cells were transfected with either scrambled siRNA or beclin 1-targeting siRNA using interfering siRNA transfection reagent (PolyPlus Transfection SA, Illkirch, France) according to the recommendations of manufacturer. The si-BECN1 targeting sequence was UUC AAC ACU CUU CAG CUC AUC AUC C and the scrambled siRNA sequence was UUC UCC GAA CGU GUC ACG UTT. All siRNAs were obtained from GenePharma, Ltd. (Shanghai, China).

Statistical analyses. To determine whether the difference between 2 groups was statistically significant, Student's t-tests were performed. The significance level was set at $\mathrm{P} \leq 0.05$.

\section{Results}

Gemcitabine decreases cell viability and induces apoptosis in MDA-MB-231 cells. The MDA-MB-231 cells were treated with gemcitabine at various concentrations, and cell viability was assessed with by MTT assay. The results revealed that cell viability (\% of the control) gradually decreased with the increasing concentrations of gemcitabine (Fig. 1B). The half maximal inhibitory concentration $\left(\mathrm{IC}_{50}\right)$ of $48 \mathrm{~h}$ of treatment with gemcitabine in the MDA-MB-231 cells was $1.2 \mu \mathrm{g} / \mathrm{ml}$. In this study, $1 \mu \mathrm{g} / \mathrm{ml}$ was selected as the treatment concentration in the subsequent experiments. Flow cytometry with AV-FITC/ PI staining was used to determine the apoptotic rate of the MDA-MB-231 cells following treatment with gemcitabine for different periods of time. The AV-FITC-positive cells were defined as apoptotic and double-negative cells were considered viable. The percentage of AV-FITC-positive cells increased with the increasing duration of gemcitabine treatment (Fig. 1C).

Gemcitabine induces mTOR-independent autophagy in triple-negative $M D A-M B-231$ cells. To evaluate gemcitabine-induced autophagy, we first tested the conversion of LC3-I into LC3-II in the MDA-MB-231 cells. As shown in Fig. 2A, gemcitabine induced the conversion of LC3-I into LC3-II in a time-dependent manner. Compared with that at $24 \mathrm{~h}$, the ratio of LC3-II/ $\beta$-actin at $48 \mathrm{~h}$ was decreased, possibly due to the fact that autophagy is a highly dynamic, multi-step process. We further examined the autophagic flux by monitoring the protein level of LC3-II relative to $\beta$-actin after $24 \mathrm{~h}$ of treatment with gemcitabine with or without chloroquine (CQ) (Fig. 2B). LC3-II protein expression was markedly increased following treatment with $\mathrm{CQ}$, and the relative level of LC3-II vs. actin was significantly higher than that observed with CQ treatment alone following treatment with gemcitabine plus CQ. No apparent change in the mTOR protein levels was observed following treatment with 
A

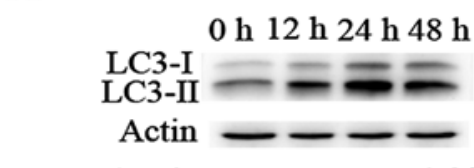

LC3-II/Actin 0.681 .191 .661 .28
B

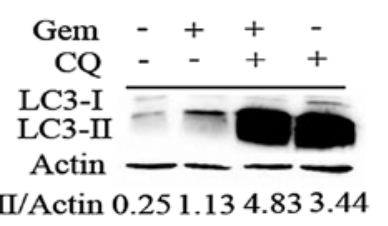

C $\begin{array}{rllll}\mathrm{Gem}(\mu \mathrm{g} / \mathrm{ml}) & 0 & 0.5 & 1 & 2 \\ \mathrm{mTOR} & - & - & - \\ \text { Actin } & - & - & -\end{array}$
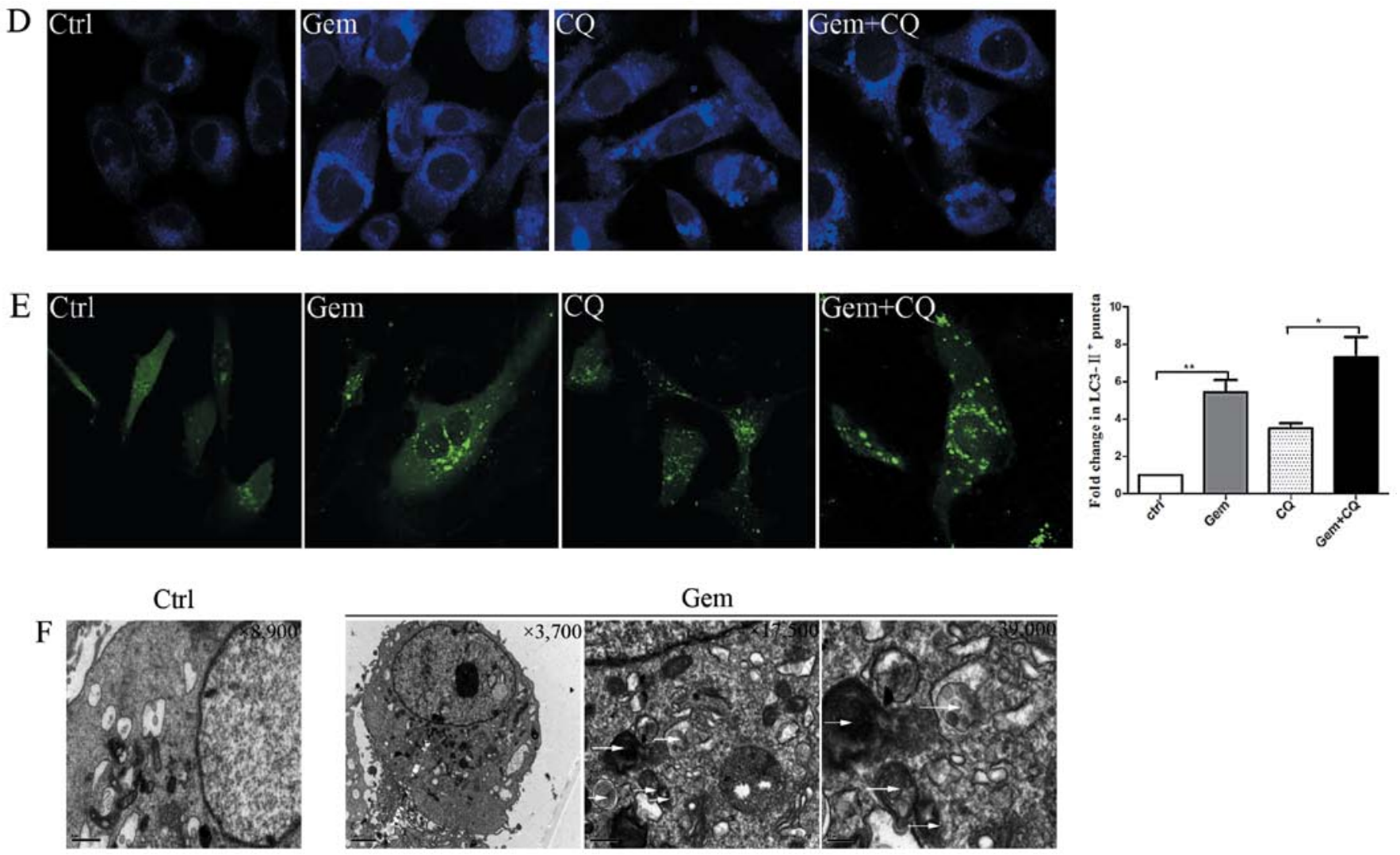

Gem

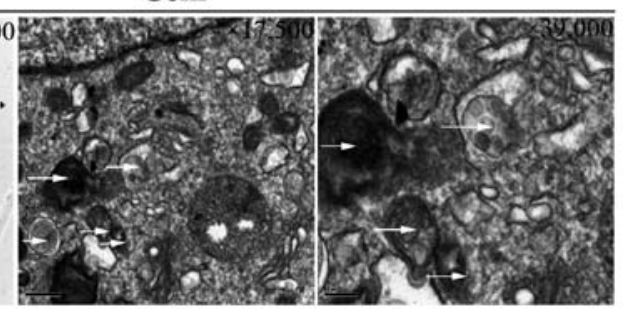

Figure 2. Gemcitabine induces mTOR-independent autophagy in triple-negative MDA-MB-231 cells. (A) The conversion of Lc3-I (18 KDa) into LC3-II (16 KDa) was assayed in cells treated with gemcitabine $(1 \mu \mathrm{g} / \mathrm{ml})$ for $0 \mathrm{~h}$ (untreated control) or 12, 24 and $48 \mathrm{~h}$. (B) Cells were treated with gemcitabine, chloroquine (CQ; $5 \mu \mathrm{M}$; added prior to treatment with gemcitabine for $1 \mathrm{~h}$ ), or gemcitabine plus CQ for $0 \mathrm{~h}$ (control) or $24 \mathrm{~h}$, then the conversion of Lc3-I into LC3-II was assessed. (C) The levels of mTOR protein were assessed following treatment with gemcitabine (0,0.5 and $1,2 \mu \mathrm{g} / \mathrm{ml})$ for $24 \mathrm{~h}$. (D) Cells exposed to gemcitabine, CQ, or gemcitabine plus CQ for $0 \mathrm{~h}$ (control) or $24 \mathrm{~h}$ were treated with monodansylcadaverine (MDC) (50 $\mu$ mol/1) for 15 min and the samples were examined under a confocal microscope. (E) Cells transiently transfected with EGFP-LC3 plasmids were exposed to gemcitabine, CQ, or gemcitabine plus CQ for 0 h (control) or $24 \mathrm{~h}$. A confocal microscope was used to measure EGFP fluorescence distribution. The punctate EGFP-LC3 was indicative of autophagosomes. The number of LC3-II' puncta was counted in at least 75 cells from random fields and the fold change was calculated by normalizing to the amount of the control group. (F) Cells were treated with gemcitabine for 0 (control) or $24 \mathrm{~h}$. The ultrastructural characteristics of the cells were analyzed by transmission electron microscopy (TEM). Autophagical vacuoles with typical double-layer membrane containing remnants of organelles are highlighted by white arrows. $\mathrm{P}<0.05$; $^{* *} \mathrm{P}<0.01$. Gem, gemcitabine; Ctrl, control.

various concentrations of gemcitabine, indicating that the gemcitabine-induced autophagy in MDA-MB-231 cells was possibly mTOR-independent (Fig. 2C).

MDC is a selective fluorescent dye used to observe for autophagic vacuoles. As shown in Fig. 2D, gemcitabine treatment increased the number of bright blue puncta. Moreover, the number of MDC-labeled puncta was markedly increased and the average puncta volume was significantly larger following treatment with gemcitabine plus CQ. The transient transfection of EGFP-LC3 plasmids was performed to further determine gemcitabine-induced autophagy in the MDA-MB-231 cells. The number of EGFP-LC3 puncta per cell in the group treated with gemcitabine plus CQ was markedly higher than that observed in the cells treated with gemcitabine alone or $\mathrm{CQ}$ alone (Fig. 2E), thus indicating the increased autophagic flux level in triple-negative MDA-MB-231 cells treated with gemcitabine. Finally, TEM confirmed that numerous autophagic vacuoles with a typical double-layer membrane containing organelle remnants were present in the gemcitabine-treated MDA-MB-231 cells as opposed to the untreated (control) cells (Fig. 2F). Our results indicated that gemcitabine treatment induce autophagy in TNBC cells.

Gemcitabine-induced autophagy plays a cytoprotective role in triple-negative $M D A-M B-231$ cells. As shown by the results of MTT assay, CQ treatment alone $(5 \mu \mathrm{M})$ had little toxic effect on the MDA-MB-231 cells (Fig. 3A). However, the inhibition of autophagy by CQ significantly enhanced the inhibitory effects of gemcitabine on cell viability at 12 and 48 h (Fig. 3B). Flow cytometry revealed that after the cells 


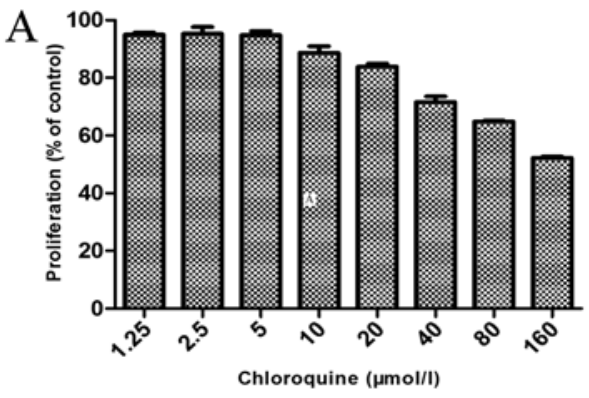

$\mathrm{C}$

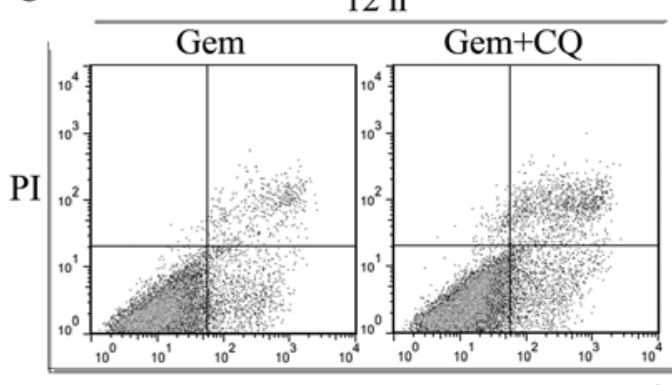

B
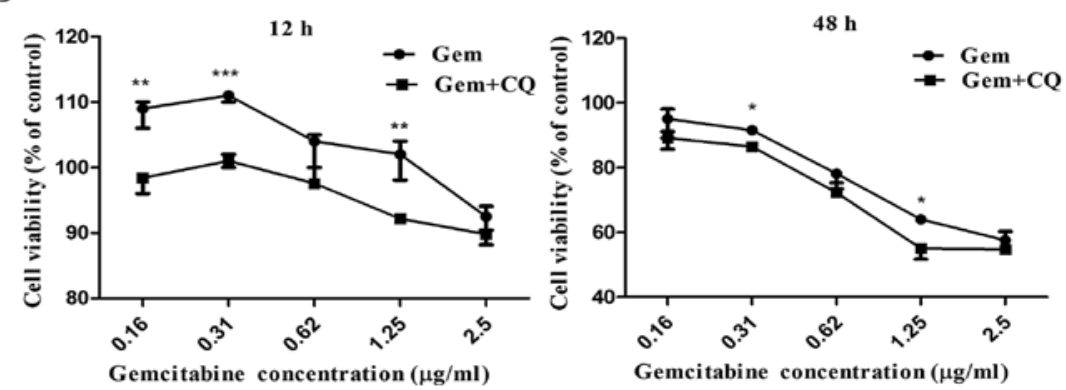

Annexin V-FITC

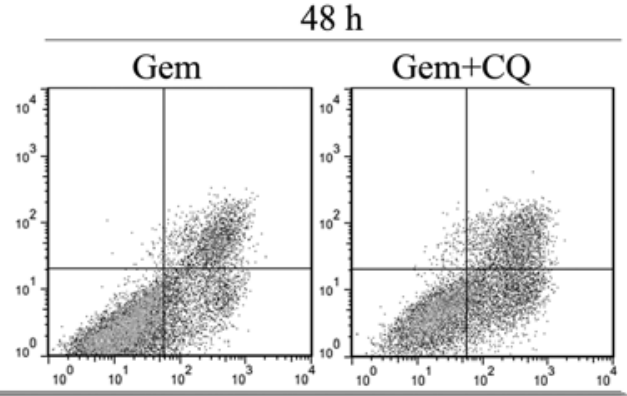

$\mathrm{Gem}+\mathrm{CQ}$



Figure 3. Inhibition of autophagy by chloroquine (CQ) enhances the cytotoxicity of gemcitabine in triple-negative MDA-MB-231 cells. (A) The effect of chloroquine $(1.25-160 \mu \mathrm{mol} / 1)$ for $48 \mathrm{~h}$ on MDA-MB-231 cell growth was determined by MTT assay. The results are expressed as the relative percentage of each group compared with the control. (B) The viability of MDA-MB-231 cells exposed to gemcitabine alone $(0.16-2.5 \mu \mathrm{g} / \mathrm{ml})$ or gemcitabine plus CQ (5 $\mu$ M; added prior to treatment with gemcitabine for $1 \mathrm{~h}$ ) for 12 or $48 \mathrm{~h}$ was determined by MTT assay. The results are expressed as the relative percentage of each group compared with the control. The data [means \pm standard error (SE)] were from 3 independent experiments. (C) Apoptosis was measured by Annexin V-FITC (AV-FITC)/ propidium iodide (PI) staining and flow cytometry following treatment with gemcitabine $(1 \mu \mathrm{g} / \mathrm{ml})$ or gemcitabine plus CQ for 12 or $48 \mathrm{~h}$. The scatter plots and the statistical analysis of Annexin V-FITC and PI uptake are shown. ${ }^{*} \mathrm{P}<0.05 ;{ }^{* *} \mathrm{P}<0.01 ;{ }^{* * *} \mathrm{P}<0.001$. ns, not significant; Gem, gemcitabine.

were treated with gemcitabine plus CQ, the number of apoptotic markedly increased compared with the cells treated with gemcitabine alone (Fig. 3C).

We further inhibited autophagy by silencing the beclin 1 gene, as beclin 1 is one of the essential autophagy-related genes (Atgs) (26). Transfection with beclin 1-targeting siRNA resulted in a marked reduction in beclin 1 protein expression, while the scrambled siRNA-transfected group showed no change (Fig. 4A). As shown in Fig. 4B, beclin 1 knockdown markedly reduced the viability of cells treated with gemcitabine at 12 and $48 \mathrm{~h}$. Of note, si-BECN1 alone also inhibited cell viability, thus indicating that beclin 1 plays a positive role in the growth of TNBC cells. As shown by flow cytometry, treatment with scrambled siRNA plus gemcitabine produced results that were comparable to those that were obtained by treatment with gemcitabine alone, while treatment with gemcitabine plus si-BECN1 led to a more significant increase in the percentage of apoptotic cells (Fig. 4C). These results demonstrate that beclin 1-mediated autophagy induced by gemcitabine contributes to TNBC cell growth and survival.

Autophagy inhibition by CQ shifts the expression levels of 553 and Bcl-2 family proteins in favor of promoting apoptosis. The p53 and Bcl-2 family member proteins play a critical role in the intrinsic apoptotic pathway, which regulates apoptosis by modulating mitochondrial outer membrane integrity (27).

We investigated the protein levels of $\mathrm{p} 53$, the anti-apoptotic $\mathrm{Bcl}-\mathrm{xL}$ gene and the pro-apoptotic Bax gene. As shown by our results, the $\mathrm{Bcl}-\mathrm{xL}$ protein levels increased, while the p53 and
Bax protein levels decreased in a concentration-dependent manner following treatment with gemcitabine (Fig. 5A, left panel). Following treatment with gemcitabine plus the autophagic inhibitor, $\mathrm{CQ}$, the Bcl-xL protein levels markedly decreased with a concomitant increase in the p53 and proapoptotic Bax levels in a concentration-dependent manner, which was in contrast to what was observed following treatment with gemcitabine alone (Fig. 5A, right panel). We further assessed the relative $\mathrm{Bax} / \mathrm{Bcl}-\mathrm{xL}$ ratio following treatment with gemcitabine with or without CQ. With the increasing gemcitabine concentration, the relative $\mathrm{Bax} / \mathrm{Bcl}-\mathrm{xL}$ ratio slightly decreased, while it markedly increased following treatment with CQ plus gemcitabine (Fig. 5B).

\section{Discussion}

A number of studies have demonstrated that anti-cancer therapy induces autophagy in cancer cells, while there is an ongoing debate on the association between autophagy and therapeutic efficacy (28). A series of studies have reported that chemotherapeutic drugs induce autophagy, promoting the survival of cancer cells (29-31), thus suggesting that autophagy is involved in the development of resistance to chemotherapy.

CQ has been extensively used as an autophagic inhibitor that prevents the acidification of the lysosome, thus blocking the late stage of the autophagy process (32). It is noteworthy to mention that some clinical trials testing CQ-mediated autophagy inhibition, using hydroxychloroquine (HCQ) combined with ixabepilone for metastatic breast cancer and CQ for ductal 
A

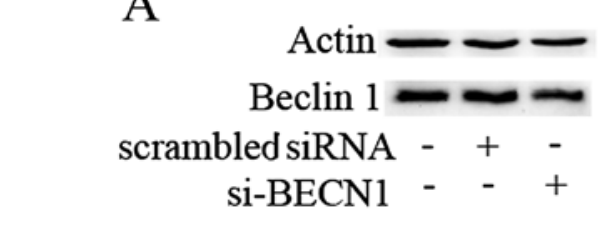

B

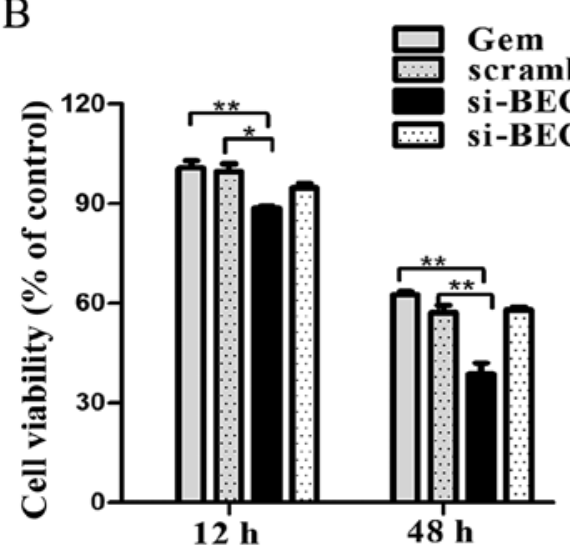

$\mathrm{C}$

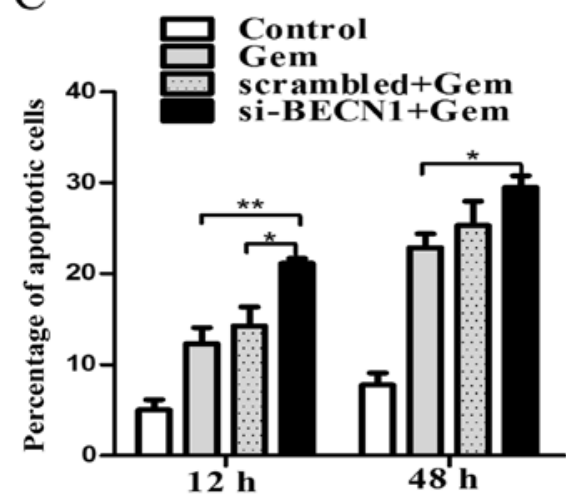

Figure 4. Inhibition of autophagy by si-BECN1 enhances the efficacy of gemcitabine in triple-negative MDA-MB-231 cells. (A) The levels of beclin 1 protein were detected following treatment with scrambled siRNA or si-BECN1 for $48 \mathrm{~h}$. (B) Cells were treated with scrambled siRNA or si-BECN1 for $48 \mathrm{~h}$, and then incubated with or without gemcitabine $(1 \mu \mathrm{g} / \mathrm{ml})$ for 12 or $48 \mathrm{~h}$ Cell viability was measured by MTT assay and the results are expressed as the relative percentage of each group compared with the control. The results [means \pm standard error (SE)] were from 3 independent experiments. (C) Cells were treated with or without scramble siRNA or si-BECN1 for $48 \mathrm{~h}$, followed by treatment with gemcitabine for 12 or $48 \mathrm{~h}$, and apoptosis was determined by Annexin V-FITC (AV-FITC)/propidium iodide (PI) staining and flow cytometry. The results (means $\pm \mathrm{SE}$ ) were from 3 independent experiments. ${ }^{*} \mathrm{P}<0.05$; ${ }^{* *} \mathrm{P}<0.01$. Gem, gemcitabine.

carcinoma in situ, have been carried out (http://www.clinical trials.gov/ct2/show/results/NCT00765765) or are ongoing (http://www.clinicaltrials.gov/ct2/show/NCT01023477); this indicates that chloroquine is clinically applicable. Our results demonstrated that the addition of CQ to gemcitabine treatment greatly enhanced the inhibitory effect on cell viability and increased cell apoptosis in the MDA-MB-231 cells. Our data support the therapeutic potential of CQ in combination with gemcitabine for enhancing the efficacy of gemcitabine in TNBC.

Beclin 1 is an essential Atg that signals the onset of the autophagy process (33). In our study, the silencing of beclin 1
A

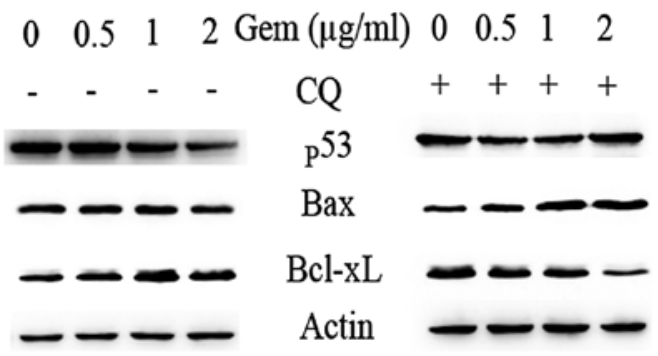

B

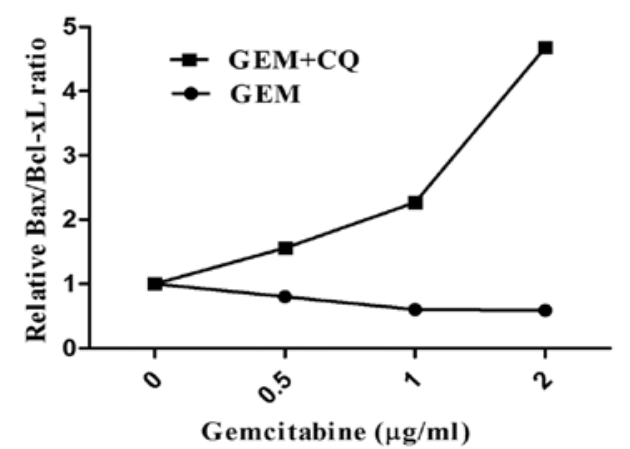

Figure 5. Autophagy inhibition by chloroquine (CQ) shifts the expression of the $\mathrm{p} 53$ and $\mathrm{Bcl}-2$ family proteins in favor of promoting apoptosis. (A) p53, Bax and Bcl-xL protein levels were detected following treatment with gemcitabine $(0,0.5,1$ and $2 \mu \mathrm{g} / \mathrm{ml})$ or gemcitabine plus CQ $(5 \mu \mathrm{M}$, added prior to treatment with gemcitabine for $1 \mathrm{~h})$. (B) The fold change of the Bax/Bcl-xL ratio relative to the control group with the increasing gemcitabine concentration was calculated. Gem, gemcitabine.

significantly enhanced the inhibitory effect of gemcitabine on the growth and viability of MDA-MB-231 cells, indicating that beclin 1-mediated autophagy plays a cytoprotective role in TNBC cells. Of note, treatment with si-BECN1 alone markedly inhibited the viability of MDA-MB-231 cells, indicating that the beclin 1 gene plays a positive role in the growth of TNBC cells. By contrast, the study by Liang et al (26) produced different results. They evaluated the effects of beclin 1 on the growth of $\mathrm{ER}^{+} \mathrm{MCF}-7$ cells before and after stably transfecting MCF-7 cells with a vector containing flag-beclin 1 . They also evaluated cell proliferation and tumor formation in nude mice with MCF-7 tumors. The number of MCF-7 beclin 1 clones was significantly lower compared with the control group. Therefore, the authors concluded that beclin 1 acts as a negative regulator of mammary cell growth and tumorigenesis (26). Thus, it can be hypothesized that the effects of beclin 1 on the growth of breast cancer cells may depend on the status of steroid hormone receptors.

There are multiple connections between the apoptotic and autophagic processes (21). In this study, we demonstrated that pre-treatment with CQ (to inhibit autophagy) prior to gemcitabine treatment shifted the p53 protein level and the $\mathrm{Bax} / \mathrm{Bcl}-\mathrm{xL}$ ratio in favor of promoting apoptosis. This indicates that gemcitabine-induced autophagy inhibits the apoptotic process to a certain extent, and that the inhibition of the induced autophagy may promote apoptosis, resulting in an improved efficacy of gemcitabine in TNBC cells. Our results also suggest that the inhibitory effect of autophagy on apoptosis is another mechanism behind autophagy-induced 
cytoprotection other than the basic cellular functions of autophagy in triple-negative MDA-MB-231 cells.

In conclusion, the present study clearly demonstrates that gemcitabine induces mTOR-independent autophagy in triple-negative MDA-MB-231 cells and suggests that gemcitabine-induced autophagy plays an active role in the refractory response of TNBC cells to gemcitabine. Our results also indicate that the downregulation of cellular apoptotic levels by autophagy constitutes another mechanism responsible for autophagy-induced cytoprotection in gemcitabine-exposed TNBC cells. Thus, the combination of an autophagic inhibitor with gemcitabine may be a promising approach to promote greater therapeutic efficacy in patients with TNBC. However, more preclinical trials are required to further determine the positive effects of autophagic inhibitors on gemcitabine treatment in patients with TNBC.

\section{Acknowledgements}

We gratefully acknowledge the help of Professor Wang Xiaojian (The Institute of Molecular Immunology, Zhejiang University, Hangzhou, China) for kindly providing the reagents.

\section{References}

1. Siegel R, Naishadham D and Jemal A: Cancer statistics, 2013. CA Cancer J Clin 63: 11-30, 2013.

2. Irvin WJ Jr and Carey LA: What is triple-negative breast cancer? Eur J Cancer 44: 2799-2805, 2008.

3. Bauer KR, Brown M, Cress RD, Parise CA and Caggiano V: Descriptive analysis of estrogen receptor (ER)-negative, progesterone receptor (PR)-negative, and HER2-negative invasive breast cancer, the so-called triple-negative phenotype: a population-based study from the California cancer Registry. Cancer 109: 1721-1728, 2007.

4. Lin NU, Claus E, Sohl J, Razzak AR, Arnaout A and Winer EP: Sites of distant relapse and clinical outcomes in patients with metastatic triple-negative breast cancer: high incidence of central nervous system metastases. Cancer 113: 2638-2645, 2008.

5. Haffty BG, Yang Q, Reiss M, et al: Locoregional relapse and distant metastasis in conservatively managed triple negative early-stage breast cancer. J Clin Oncol 24: 5652-5657, 2006.

6. Dent R, Trudeau M, Pritchard KI, et al: Triple-negative breast cancer: clinical features and patterns of recurrence. Clin Cancer Res 13: 4429-4434, 2007.

7. Kassam F, Enright K, Dent R, et al: Survival outcomes for patients with metastatic triple-negative breast cancer: implications for clinical practice and trial design. Clin Breast Cancer 9: 29-33, 2009.

8. Huang P, Chubb S, Hertel LW, Grindey GB and Plunkett W: Action of 2',2'-difluorodeoxycytidine on DNA synthesis. Cancer Res 51: 6110-6117, 1991.

9. Silvestris N, D'Aprile M, Andreola G, Locopo N, Marini L, Crucitta E, De Lena M and Lorusso V: Rationale for the use of gemcitabine in breast cancer (Review). Int J Oncol 24: 389-398, 2004.

10. Passardi A, Massa I, Zoli W, et al: Phase II study of gemcitabine, doxorubicin and paclitaxel (GAT) as first-line chemotherapy for metastatic breast cancer: a translational research experience. BMC Cancer 6: 76, 2006.

11. O'Shaughnessy JA, Pluenneke R, Sternberg J, Khandelwal P, Ilegbodu D and Asmar L: Phase II trial of weekly docetaxel/ gemcitabine as first-line chemotherapy in patients with locally recurrent or metastatic breast cancer. Clin Breast Cancer 6 : 505-510, 2006.
12. Tomao S, Romiti A, Tomao F, et al: A phase II trial of a biweekly combination of paclitaxel and gemcitabine in metastatic breast cancer. BMC Cancer 6: 137, 2006.

13. Hernández-Vargas H, Rodríguez-Pinilla SM, Julián-Tendero M, et al: Gene expression profiling of breast cancer cells in response to gemcitabine: NF-kappaB pathway activation as a potential mechanism of resistance. Breast Cancer Res Treat 102: 157-172, 2007.

14. Mizushima N and Komatsu M: Autophagy: renovation of cells and tissues. Cell 147: 728-741, 2011.

15. Rubinsztein DC, Codogno P and Levine B: Autophagy modulation as a potential therapeutic target for diverse diseases. Nat Rev Drug Discov 11: 709-730, 2012.

16. Kroemer G and Levine B: Autophagic cell death: the story of a misnomer. Nat Rev Mol Cell Biol 9: 1004-1010, 2008.

17. Baehrecke EH: Autophagy: dual roles in life and death? Nat Rev Mol Cell Biol 6: 505-510, 2005.

18. Levine B and Yuan J: Autophagy in cell death: an innocent convict? J Clin Invest 115: 2679-2688, 2005.

19. Kroemer G and Jäättelä M: Lysosomes and autophagy in cell death control. Nat Rev Cancer 5: 886-897, 2005.

20. Gozuacik D and Kimchi A: Autophagy and cell death. Curr Top Dev Biol 78: 217-245, 2007.

21. Maiuri MC, Zalckvar E, Kimchi A and Kroemer G: Self-eating and self-killing: crosstalk between autophagy and apoptosis. Nat Rev Mol Cell Biol 8: 741-752, 2007.

22. Donohue E, Thomas A, Maurer N, et al: The autophagy inhibitor verteporfin moderately enhances the antitumor activity of gemcitabine in a pancreatic ductal adenocarcinoma model. J Cancer 4: 585-596, 2013.

23. Pardo R, Lo Ré A, Archange C, et al: Gemcitabine induces the VMP1-mediated autophagy pathway to promote apoptotic death in human pancreatic cancer cells. Pancreatology 10: 19-26, 2010.

24. Mukubou H, Tsujimura T, Sasaki R and $\mathrm{Ku} \mathrm{Y}$ : The role of autophagy in the treatment of pancreatic cancer with gemcitabine and ionizing radiation. Int J Oncol 37: 821-828, 2010.

25. Papademetrio DL, Cavaliere V, Simunovich T, et al: Interplay between autophagy and apoptosis in pancreatic tumors in response to gemcitabine. Target Oncol: Apr 16, 2013 (Epub ahead of print)

26. Liang XH, Jackson S, Seaman M, et al: Induction of autophagy and inhibition of tumorigenesis by Beclin 1. Nature 402: 672-676, 1999.

27. Jain MV, Paczulla AM, Klonisch T, et al: Interconnections between apoptotic, autophagic and necrotic pathways: implications for cancer therapy development. J Cell Mol Med 17: 12-29, 2013.

28. Kondo Y, Kanzawa T, Sawaya R and Kondo S: The role of autophagy in cancer development and response to therapy. Nat Rev Cancer 5: 726-734, 2005.

29. Li J, Hou N, Faried A, Tsutsumi S and Kuwano H: Inhibition of autophagy augments 5-fluorouracil chemotherapy in human colon cancer in vitro and in vivo model. Eur J Cancer 46: 1900-1909, 2010.

30. Liu D, Yang Y, Liu Q and Wang J: Inhibition of autophagy by 3-MA potentiates cisplatin-induced apoptosis in esophageal squamous cell carcinoma cells. Med Oncol 28: 105-111, 2011.

31. Sun WL, Chen J, Wang YP and Zheng H: Autophagy protects breast cancer cells from epirubicin-induced apoptosis and facilitates epirubicin-resistance development. Autophagy 7: 1035-1044, 2011.

32. Carew JS, Medina EC, Esquivel JA II, et al: Autophagy inhibition enhances vorinostat-induced apoptosis via ubiquitinated protein accumulation. J Cell Mol Med 14: 2448-2459, 2010.

33. Klionsky DJ, Abdalla FC, Abeliovich H, et al: Guidelines for the use and interpretation of assays for monitoring autophagy. Autophagy 8: 445-544, 2012. 